\title{
An imprecise path to precision medicine
}

\author{
Xiling Shen is the Hawkins Family Associate Professor of Biomedical Engineering and director of the Woo Center \\ for Big Data and Precision Health at Duke University. He is a National Science Foundation early-career awardee, \\ chair of the National Cancer Institute's Patient-Derived Models of Cancer consortium and an Israeli faculty fellow.
}

\section{Xiling Shen}

M y mother wanted me to be a doctor, and I was sure of it until high school, when my uncle-inlaw suddenly fell ill and kept coughing and losing weight. He was treated for pneumonia until 4 months later, when he was diagnosed with colon-cancer lung metastasis. How can medicine be so imprecise? How could the doctors not know? Seeing him wasting away, suffering tremendous pain and begging to end his life, while at his last moment, his burst of every bit remaining in him to cling to life, was too much to bear. I told my mother that I could never be a doctor and face such suffering, and I later chose to be an electrical engineer so I only needed to deal with machines, without any emotional toll.

I was a successful electrical engineer, finishing my PhD at Stanford in 2005, when my advisor was diagnosed with kidney cancer. Fortunately, he was diagnosed early enough to be cured. We had long discussions on how complex biological circuits can go wrong, and whether what we learned from electrical circuits can shed some light. I started to use engineering tools to model biological circuits and found that many seemingly 'trivial' or 'redundant' features are essential for robustness or prevent the circuit from malfunctioning. Subversions of such features can give rise to diseases like cancer.

After I became an electrical engineering professor at Cornell University in 2009, I was approached by a clinical geneticist who specialized in colorectal cancer, after a talk that I gave that had touched on the circuits that regulate the cell cycle. He asked, "It's fascinating to know how the regulatory circuits can break down in a million ways and give rise to cancer, but how can we fix them?" It sounded very daunting back then. How can we know how the circuit is malfunctioning in each patient? I wondered how I could model each patient's cancer 'circuitry' and modulate it to know how to fix it. It seemed that I had better stick to conventional electrical engineering, where every parameter can be precisely measured and tested.

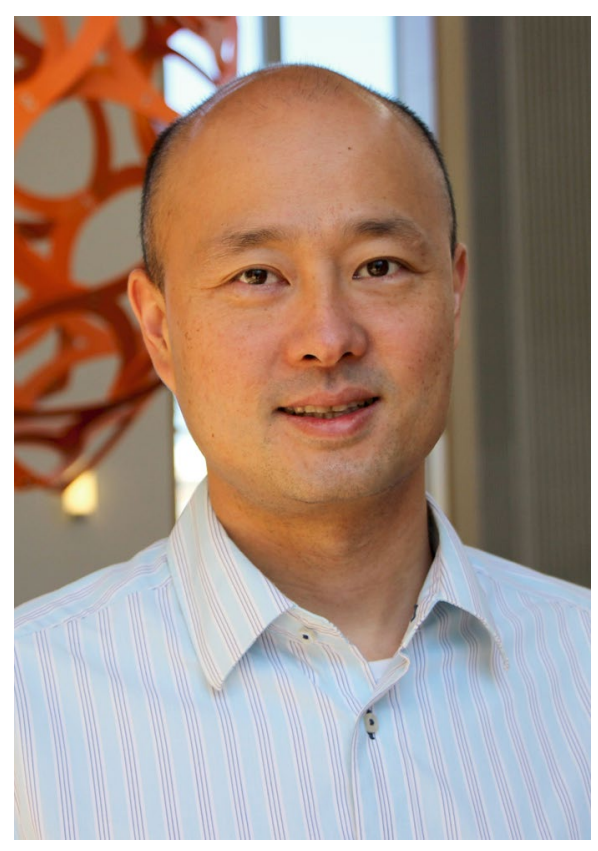

Credit: Michaela Kane/Duke University

Fortunately, 10 years ago we witnessed an acceleration in genomic sequencing and new gene-editing technologies that has allowed us to measure aberrations in the biological circuit and how its safeguards break down at the DNA level in each individual patient, at unprecedented resolution. It became possible to develop personalized therapy based on this knowledge.

A key moment came for me in 2009, when I saw the first paper from Hans Clevers' lab describing 3D organoids, a new technology that allows us to model and manipulate primary patient tissues. It dawned on me that this was the last missing piece to solving the puzzle-now we could not only make precise genomic measurements, we also had living tissue assays for functional testing. The age of precision medicine powered by big data had arrived! I switched my entire lab to study precision medicine.
Four years ago, I was visiting Duke University, when I met David Hsu, a medical oncologist. After learning about my work, he asked, "Why are you not designing a new organoid-based clinical trial to make a real impact?" This vision captivated me. The image of my uncle-in-law came back to me-maybe I could do something to help many cancer patients like him. I moved my lab to Duke and started to work with Dr. Hsu. It was a long journal to start precision cancer therapy clinical trials, as no pharma companies will fund a clinical trial that does not test their drug exclusively. However, our persistence impressed Sherry and John Woo, visionary philanthropists, who endowed the Woo Center for Big Data and Precision Health. This alternative funding source allowed us to design innovative clinical trials that take advantage of cutting-edge engineering and data technology to bring precision therapy based on big data to individual patients. The first clinical trial was approved in September 2019 , and patient recruitment is starting in January 2020.

Precision medicine requires big data, which is inherently noisy and full of unpredictability. As with many people in this new field, my serendipitous path to big data and precision health was full of unexpected turns. However, seemingly irrelevant experiences and skills picked up along our fortuitous journey end up making each of us unique and contribute to our successevery turning point turns out to be a missing piece that, together, helps us solve a gigantic puzzle.

\section{Xiling Shen}

Department of Biomedical Engineering, Woo Center for Big Data and Precision Health, Duke University, Durham, NC, USA.

e-mail:xiling.shen@duke.edu

Published online: 13 January 2020 https://doi.org/10.1038/s41591-019-0718-6 